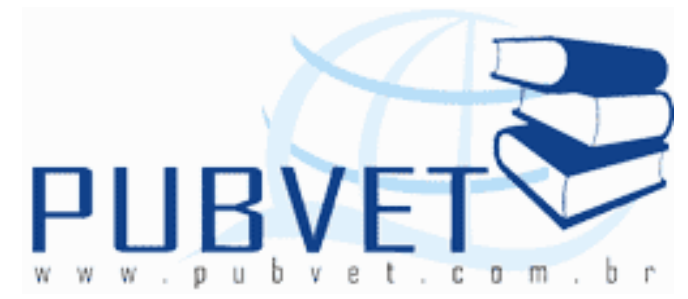

PUBVET, Publicações em Medicina Veterinária e Zootecnia.

\title{
Inviabilidade do desenvolvimento de potros após prenhez múltipla
}

$\overline{\text { David Baruc Cruvinel Lima }}{ }^{1}$, Yatta Linhares Boakari², Márcia dos Santos Rizzo ${ }^{3}$,

Tânia Vasconcelos Cavalcante ${ }^{4}$, Roberta Paulo Barjud de Sousa ${ }^{5}$, Camila Arrivabene Neves ${ }^{1}$, Mônica Arrivabene ${ }^{6}$

${ }^{1}$ Acadêmico de Medicina Veterinária - Centro de Ciências Agrárias (CCA) Universidade Federal do Piauí (UFPI).

${ }^{2}$ Mestranda em Reprodução Animal - Universidade Estadual de São Paulo (UNESP) - Júlio de Mesquita Filho - Botucatu- SP.

${ }^{3}$ Doutora em Patologia Experimental e Comparada. Departamento de Clínica e Cirurgia - Centro de Ciências Agrárias CCA - Universidade Federal do Piauí (UFPI).

${ }^{4}$ Doutora em Medicina Veterinária. Universidade Federal do Tocantins

${ }^{5}$ Médica Veterinária Autônoma

${ }^{6}$ Doutora em Anatomia dos Animais Domésticos e Silvestres. Departamento de Clínica e Cirurgia - Centro de Ciências Agrárias (CCA) - Universidade Federal do Piauí (UFPI).

\section{Resumo}

A gestação gemelar em equinos é um evento raro na medicina veterinária e apresenta-se como desafio ao profissional veterinário. Este tipo de prenhez não é desejado, pois os conceptos normalmente são abortados ou possuem falhas no desenvolvimento fetal. $O$ objetivo deste trabalho foi relatar 0 
LIMA, D.B.C. et al. Inviabilidade do desenvolvimento de potros após prenhez múltipla. PUBVET, Londrina, V. 7, N. 11, Ed. 234, Art. 1544, Junho, 2013.

comprometimento de dois conceptos após uma gestação gemelar equina. 0 diagnóstico de gêmeos deve ser realizado no início da gestação através de ultrassonografia retal e logo após deve ser realizado o esmagamento manual de uma das vesículas embrionárias. Como são raros os casos de gêmeos, devem ser preconizados estudos a respeito do desenvolvimento de novas tecnologias visando o diagnóstico precoce e o não comprometimento do ciclo reprodutivo da égua.

Palavras-chave: prenhez, égua, gêmeos, diagnóstico.

\title{
Inviability the development of foals after multiple pregnancy
}

\begin{abstract}
Twin pregnancy in horses is rare in veterinary medicine and presents a challenge to the veterinary professional. This type of pregnancy is not desired because the fetuses are often aborted or have flaws in fetal development. The objective of this study was to report the commitment of two conceptuses after a twin gestation equine. The diagnosis of twin pregnancy should be performed at the beginning of gestation by rectal ultrasound and after should be performed the manual crushing of one of the embryonic vesicles. Since the cases of twins pregnancy are rare, studies on the development of new technologies for early diagnosis should be preconized to not impair the reproductive cycle of the mare.
\end{abstract}

Keywords: pregnancy, mare, twins, diagnosis.

\section{Introdução}

A gestação gemelar em éguas pode provocar grandes perdas econômicas para os produtores, pois seu diagnóstico e tratamento são desafiadores ao médico veterinário de equinos. O tempo de gestação de uma égua é de aproximadamente 11 meses e normalmente ocorre o nascimento de apenas um concepto. Partos gemelares podem ocorrer, porém, não se caracterizam 
LIMA, D.B.C. et al. Inviabilidade do desenvolvimento de potros após prenhez múltipla. PUBVET, Londrina, V. 7, N. 11, Ed. 234, Art. 1544, Junho, 2013.

como uma situação rotineira na reprodução de equídeos. O período de gestação na espécie equina pode ser reduzido devido à presença de mais de um feto durante a mesma gestação, sendo este período influenciado também pelo tamanho dos conceptos e das respectivas placentas (MACPHERSON \& REIMER, 2000).

A gestação de gêmeos está relacionada a uma maior incidência de distocia, fetos com desenvolvimento anormal, morte fetal, abortos espontâneos e partos prematuros quando comparados com gestações únicas. Para que o concepto possa crescer e desenvolver-se normalmente é necessário que ele seja bem nutrido durante a prenhez. Essa nutrição ocorre através de uma ligação especializada entre a fêmea e o feto por meio da placenta. (AVANTE et al., 2009; RAGGIO et al., 2008; SIDDIQUI \& EWAN, 2009).

Nos equinos não ocorre à gestação de gêmeos idênticos devido à impossibilidade da égua em obter dois conceptos a termo. Isso ocorre em função da competição entre as placentas em contato com o endométrio materno, onde o resultado é uma insuficiência placentária para a nutrição dos dois conceptos. Nestes casos pode ocorrer ou uma absorção espontânea de uma ou das duas vesículas embrionárias, ou o feto que está em desvantagem vir a óbito durante a segunda metade da gestação (GINTHER, 1986; HAFEZ \& HAFEZ, 2004).

Em razão da alta incidência de abortos e o possível comprometimento no desenvolvimento dos poucos conceptos gêmeos que sobrevivem, a gestação gemelar não é almejada pelos criadores. Em virtude do diagnóstico de gêmeos em equídeos, ocorre a indução ao aborto no início da gestação e a égua deve ser coberta novamente. O diagnóstico precoce e a utilização de técnicas que impossibilitem a continuação deste tipo de gestação colabora com a redução de abortos em éguas (HAFEZ \& HAFEZ, 2004; MACPHERSON \& REIMER, 2000).

A detecção de gestação gemelar pode ser realizada por ultrasonografia transretal através de escaneamento do corpo e cornos uterinos para identificar as possíveis vesículas embrionárias (GINTHER, 1984). Após confirmada a gestação gemelar, existem alguns procedimentos que podem ser usados para 
LIMA, D.B.C. et al. Inviabilidade do desenvolvimento de potros após prenhez múltipla. PUBVET, Londrina, V. 7, N. 11, Ed. 234, Art. 1544, Junho, 2013.

tornar a gestação única e diminuir as chances de complicações. Um dos mais comuns e eficazes é o esmagamento manual da menor vesícula embrionária até os 15 dias após a ovulação, constituindo a ruptura transretal da vesícula embrionária, permitindo o sucesso de nascimento de um potro (MOREL et al, 2012).

Diante do exposto, o objetivo deste trabalho foi relatar o comprometimento de dois conceptos após uma gestação gemelar equina, em que, após o nascimento, os potros vieram a óbito devido ao manejo errôneo na propriedade, associado ao estado nutricional deficiente dos animais.

\section{Relato de caso}

Equino, fêmea, de cinco anos de idade, de raça mestiça de Paint Horse, pesando aproximadamente $430 \mathrm{Kg}$, primípara, foi coberta por um equino macho de 11 anos, de raça Paint Horse e com peso de $450 \mathrm{Kg}$. A gestação ocorreu sem acompanhamento médico-veterinário e ao nascimento das crias foi descoberta a gestação gemelar (Figura 1), com o nascimento de um macho pesando cerca de $40 \mathrm{Kg}$, bem desenvolvido, e de uma fêmea, $20 \mathrm{Kg}$, que apresentou problemas de aprumo (Figura 2). A presença do médico veterinário somente foi requisitada após 24 horas do nascimento dos animais pelo fato do potro macho apresentar um gotejamento pelo umbigo. Após a chegada do médico veterinário constatou-se que o animal apresentava-se em um quadro de choque. 0 produto veio a óbito 48 horas após o nascimento, acometido de persistência do úraco (Figura 3 ), evoluindo com quadro de síndrome cólica e óbito. À necropsia constatou torção do colón maior (Figura 4) e septicemia. A fêmea sofreu um corte no membro anterior esquerdo na altura do codilho, evoluindo com sinais clínicos de rigidez dos membros, cauda estendida e protusão da terceira pálpebra, sobrevivendo por 110 horas após o nascimento, tendo a morte associada a uma suspeita de tétano. 
LIMA, D.B.C. et al. Inviabilidade do desenvolvimento de potros após prenhez múltipla. PUBVET, Londrina, V. 7, N. 11, Ed. 234, Art. 1544, Junho, 2013.

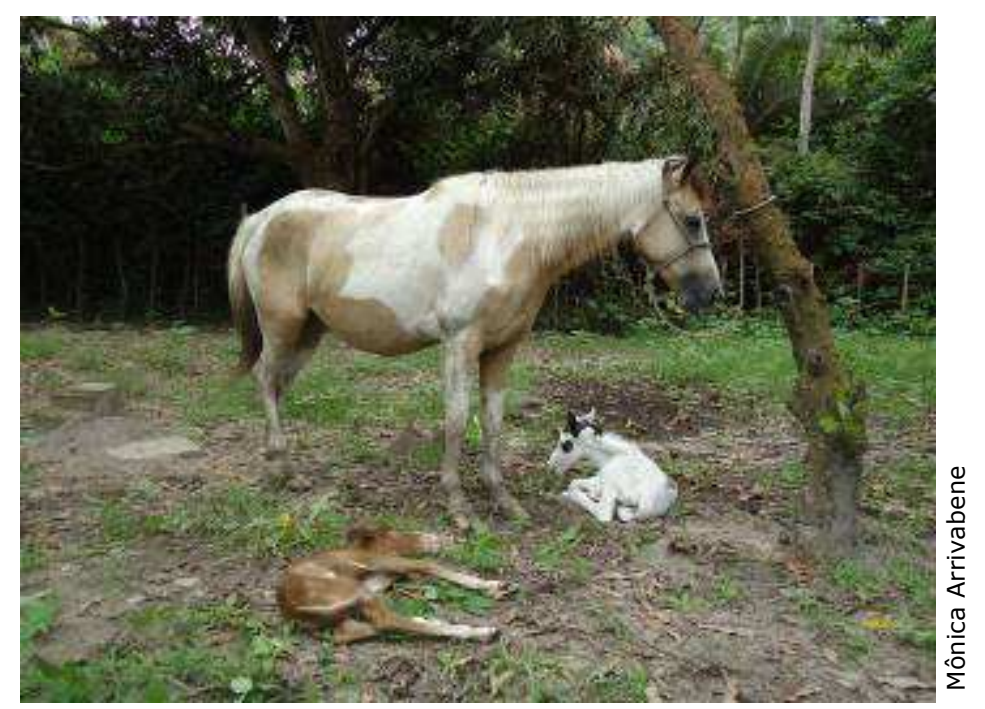

Figura 1: Fêmea a campo com os dois conceptos após o parto

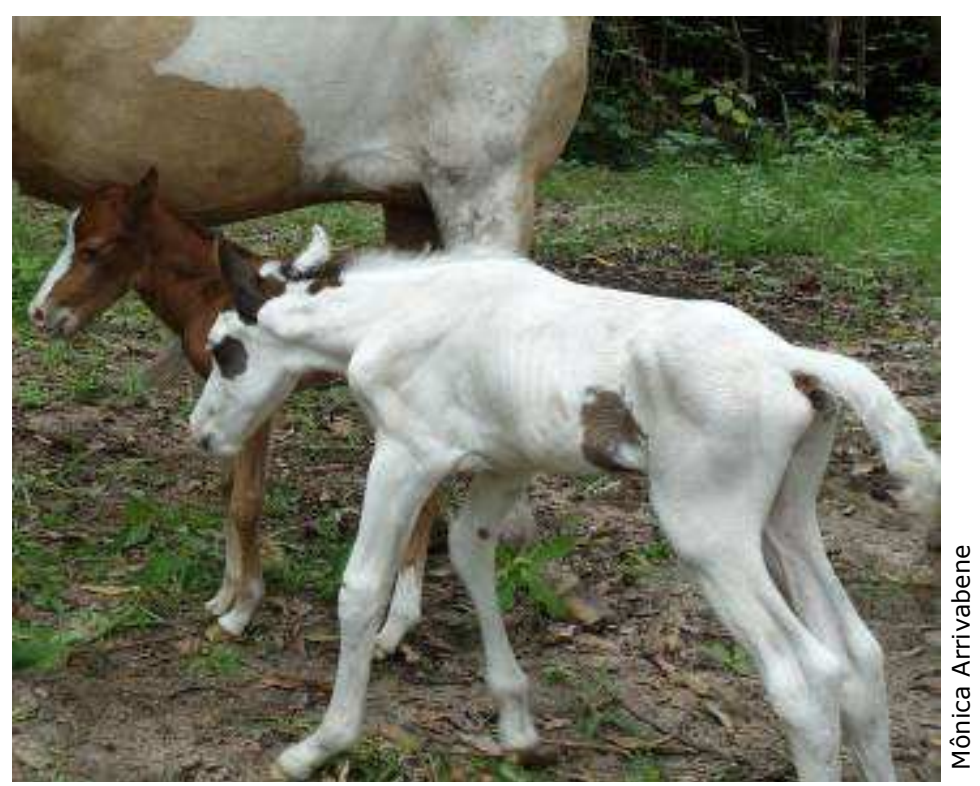

Figura 2: Potra com estado nutricional comprometido e problemas de aprumo 
LIMA, D.B.C. et al. Inviabilidade do desenvolvimento de potros após prenhez múltipla. PUBVET, Londrina, V. 7, N. 11, Ed. 234, Art. 1544, Junho, 2013.

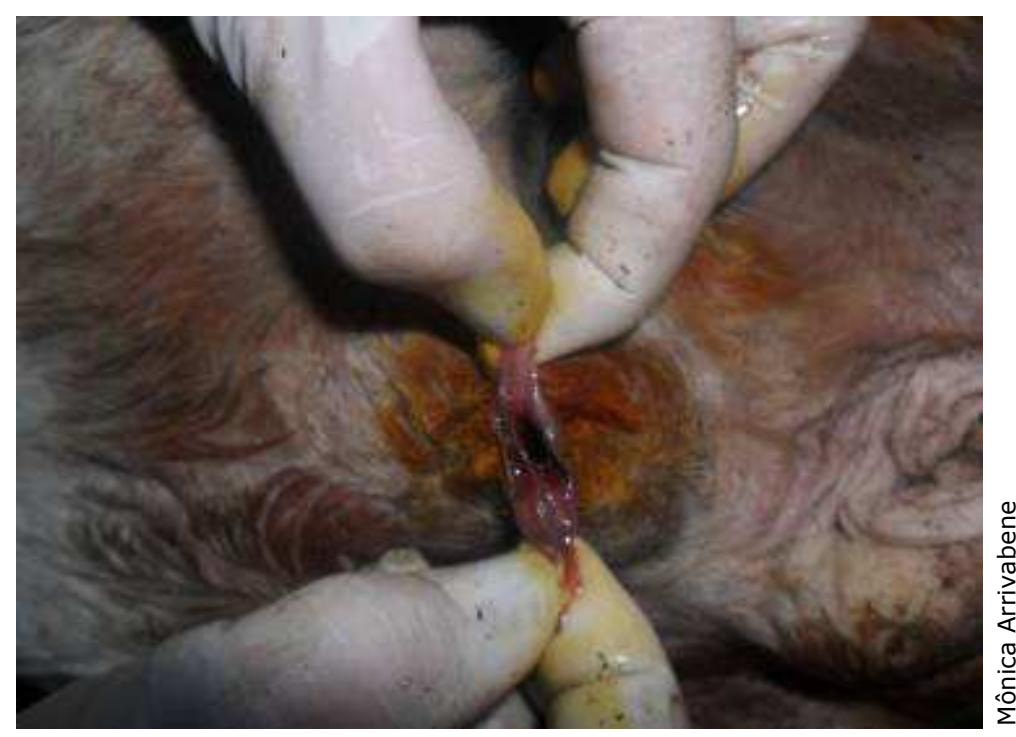

Figura 3: Persistência do Úraco no potro.

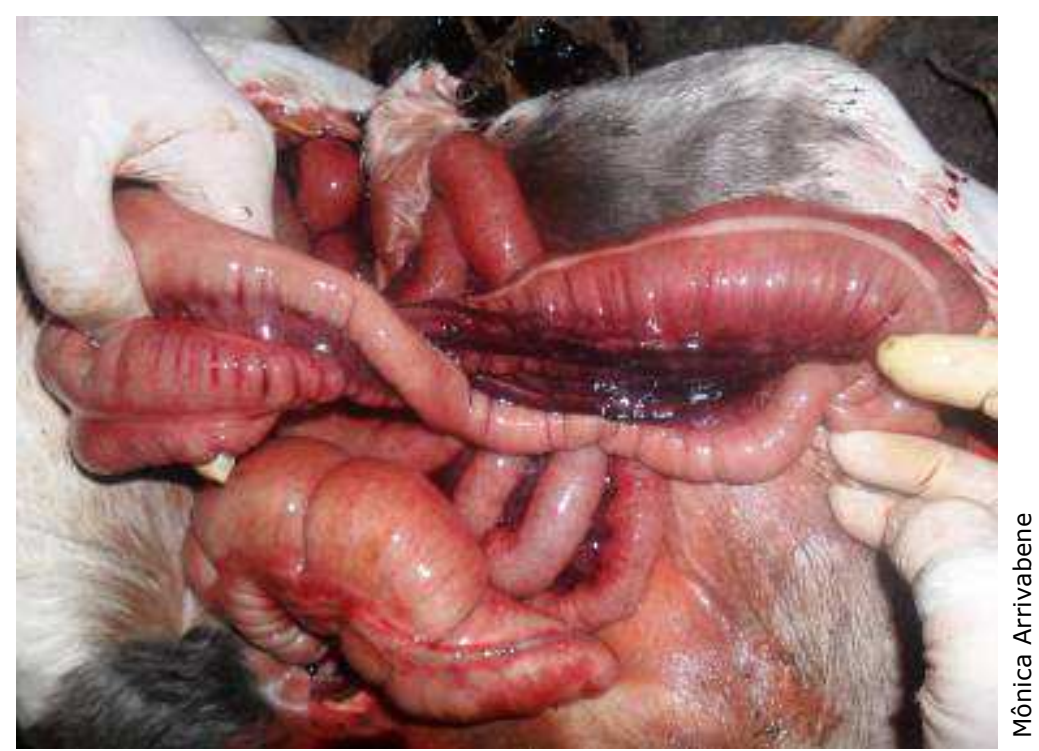

Figura 4: Torção do colón maior no potro.

A reprodução de equinos tem como objetivo a obtenção de potros com excelente estado físico e boas chances de sobrevivência, visto a reduzida prolificidade desta espécie, que normalmente é de uma cria por período (MENDES, 2011). A frequência de gêmeos em equinos é baixa $(0,5$ a $1,5 \%)$, assim, essa possibilidade é muito pequena, o que leva as gestações gemelares em equinos evoluírem para a morte embrionária ou fetal de um ou de ambos 
LIMA, D.B.C. et al. Inviabilidade do desenvolvimento de potros após prenhez múltipla. PUBVET, Londrina, V. 7, N. 11, Ed. 234, Art. 1544, Junho, 2013.

os produtos (MILLER \& WOODS, 1988; RAGGIO et al., 2008). No caso descrito, os animais vieram a óbito provavelmente por estas deficiências estarem presentes, deixando-os fracos, mal desenvolvidos, sujeitos a doenças; pela ausência de acompanhamento médico veterinário durante a gestação e o pósparto imediato e por manejo inadequado dos recém-nascidos.

Tendo em vista a criação de cavalos e a reprodução destes animais como sendo uma atividade econômica, é desejável que a égua possa parir um potro por ano e para isso é necessário que ela inicie uma nova gestação dentro de um mês após o parto. As fêmeas equinas possuem a capacidade de iniciar sua atividade ovariana e também de sofrer involução uterina precocemente e, por esse motivo, elas conseguem entrar em uma nova gestação rapidamente. Uma produção eficiente de potros se mostra como requisito primordial para a manutenção e renovação de planteis de equinos, portanto, torna-se economicamente vantajoso que a reprodução das fêmeas inicie-se o quanto antes após o parto (PAIVA, 2009). Na gestação gemelar, a égua é prejudicada em se preparar para uma nova concepção devido à incapacidade de levar a termo os conceptos e desta maneira, atrasando uma nova gestação levando a uma queda de fertilidade da égua, causando perdas econômicas aos criadores (PASCOE, 1983).

O manejo dos recém-nascidos não se apresenta como prática comum na criação de equídeos, entretanto, esta realidade necessita ser modificada, visto que o acompanhamento pós-parto pode prevenir e propiciar o diagnóstico precoce de problemas de saúde, contribuindo assim para uma diminuição na taxa de mortalidade perinatal (MENDES, 2011). Animais oriundos de gestação dupla possuem uma necessidade maior de cuidados, principalmente nas primeiras horas de vida. Os potros do caso em questão não tiveram o acompanhamento necessário nesta fase da vida, o que contribuiu para que os mesmos viessem a óbito.

As biotecnologias reprodutivas são ferramentas essenciais para o sucesso no acompanhamento reprodutivo dos equídeos, entretanto, cabe ao profissional veterinário o conhecimento de como usá-las para que se possa 
LIMA, D.B.C. et al. Inviabilidade do desenvolvimento de potros após prenhez múltipla. PUBVET, Londrina, V. 7, N. 11, Ed. 234, Art. 1544, Junho, 2013.

interpretar os dados e implementar as decisões corretas (COSTA, 2010). O acompanhamento destes profissionais durante o ciclo reprodutivo e a gestação da égua é de suma importância para evitar as perdas econômicas ocasionadas e alcançar um índice de eficiência reprodutiva satisfatória. $O$ uso de ferramentas tecnológicas por este, como a ultrassonografia, permite o diagnóstico precoce da gestação gemelar e a melhor conduta a ser adotada (MACPHERSON \& REIMER, 2000). Não houve acompanhamento médico precoce no caso descrito, o que influenciou o desempenho dos potros e as perdas econômicas ocasionadas pelo óbito de ambos conceptos.

\section{Conclusão}

A gestação gemelar em éguas é de difícil diagnóstico e se apresenta como um desafio para os profissionais de reprodução animal. Geralmente ocorre o comprometimento das crias, que acarreta em perdas econômicas aos produtores. Assim, faz-se necessário o acompanhamento médico veterinário durante todo o ciclo reprodutivo, que possibilitará a utilização de ferramentas tecnológicas diagnósticas para a orientação e execução de intervenções corretas.

\section{Referências}

AVANTE, M. L.; ZANGIROLAMI FILHO, D.; PEREIRA, D. M.; DIAS, L. G. G. G. Anexos fetais. Revista Científica Eletrônica de Medicina Veterinária, v. 7, n. 12, 2009.

COSTA, A. L. A. Controlo reprodutivo e transferência de embriões em equinos: 2010. 33 f. Dissertação (Mestrado Integrado em Medicina Veterinária) - Instituto de Ciências Biomédicas Abel Salazar, Universidade do Porto, Lisboa, 2010.

GINTHER, O. J. Intrauterine movement of the early conceptus in barren and postpartum mares. Theriogenology, v.21, n.4, p.633-644, 1984.

GINTHER, O. J. Ultrasonic imaging and reproductive events in the mare. Verlag Equiservices Cross Plains, Wisconsin, USA, 1986. 378p.

HAFEZ, B; HAFEZ, E. S. E. Reprodução animal. 7 ed. São Paulo: Manole, 2004. 513p. 
MACPHERSON, M. L.; REIMER, J. M. Twin reduction in the mare: current options. Animal Reproduction Science, v. 60, n. 61, p. 233-244, 2000.

MENDES, B. S. S. M. Caracterização e análise de alguns parâmetros produtivos e reprodutivos de um sistema extensivo de produção de poldros: 2011. 69f. Dissertação (Mestrado em Engenharia Zootécnica) - Curso de Pós-graduação em Produção Animal, Universidade Técnica de Lisboa, Lisboa, 2011.

MILLER, A.; WOODS, G. L. Diagnosis and correction of twin pregnancy in the mare. Veterinary Clinics of North America: Equine Practices, v.4, n. 2, p.215-220, 1988.

MOREL, M. C. G.; NEWCOMBE, J. R.; LAUBER, M. Manual reduction of multiple embryos in the mare: The effect on subsequent pregnancy outcome. The Veterinary Journal, v.192, n.3, p.322-325, 2012.

PAIVA, R. A. E. Avaliação da fertilidade no cio do poldro: 2009. 88f. Dissertação (Mestrado Integrado em Medicina Veterinária), Faculdade de Medicina Veterinária, Universidade Técnica de Lisboa, Lisboa, 2009.

PASCOE, R. R. Methods for the treatment of twin pregnancy in the mare. Equine Veterinary Journal, v.15, n.1, p.40-42, 1983.

PRESTES, N.C.; LANDIM-ALVARENGA, F.C. Obstetrícia Veterinária.1 ed. Rio de Janeiro: Guanabara-koogan, 2006. 241p.

RAGGIO, I.; LEFEBVRE, R. C.; POITRAS, P.; VAILLANCOURT, D.; GOFF, A. K. Twin pregnancy experimental model for transvaginal ultrasound-guided twin reduction in mares. Canadian Veterinary Journal, v.49, n.11, p.1093-1098, 2008.

SIDDIQUI, F.; EWAN, A, Mc. Twins. Obstetrics, gynaecology \& reproductive medicine, $v$. 17, n. 10, p. 289-295, 2007. 